\title{
Mortality Related Factors in Patients with Variceal Bleeding with MELD Score $\geq 18$
}

\author{
Tahir Mehmood1, Muhammad Qasim Zia², Ansar Latif3 and Saad Ansar ${ }^{4}$ \\ IDepartment of Emergency Medicine, Prince Sultan Military Medical City, Riyadh, Saudi Arabia \\ 2Department of Gastroenterology / Surgery3, Khawaja Muhammad Safdar Medical College, Sialkot, Pakistan \\ ${ }^{4}$ King Edward Medical College, Lahore, Pakistan
}

\begin{abstract}
Objective: To find and analyse the associated determinants of mortality in admitted patients in cirrhotic patients with MELD score $\geq 18$ presenting in emergency department with variceal bleeding.

Study Design: Cross-sectional study.

Place and Duration of Study: Department of Emergency Medicine, King Sultan Military Hospital Riyadh, Kingdom of Saudi Arabia, from July 2017 to January 2018.

Methodology: A total of 235 patients fulfilling the inclusion criteria were enrolled in the study. Diagnosis of cirrhosis was made if the patients had platelets $<150000 / \mu \mathrm{l}$, PT $>3 \mathrm{sec}$ (prolonged), biochemical (reversal of ALT, AST ratio, albumin $<3.5 \mathrm{~g} / \mathrm{dl}$ ) and ultrasongraphic coarse echotexture of liver and splenomegaly; and presence of all of the above variables and for at least six months. Variceal bleeding diagnosed on presentation and emergency endoscopy. MELD score was calculated by following formula. MELD $=3.78$ [Ln serum bilirubin $(\mathrm{mg} / \mathrm{dL})]+11.2[\mathrm{Ln} \mathrm{INR}]+9.57$ [Ln serum creatinine $(\mathrm{mg} / \mathrm{dl})]+6.430 \mathrm{NR}$. Outcome of patients treatment was to record associated morbidity and mortality during follow-up period of one month.

Results: There were $156(66.4 \%)$ male and 79 (33.6\%) female cirrhotic patients. The mean age was $47.8 \pm 8.7$ years. Out of 235 patients of liver cirrhosis, $47(20.0 \%)$ expired during the hospital stay, while $188(80.0 \%)$ patients survived and discharged from the hospital. Most of the cirrhotic patients were experienced with MELD score 18-20, i.e. 144 (61.3\%) followed by $70(29.8 \%)$ in $21-25$ and $21(8.9 \%)$ had the range of 26-30. In-hospital mortality rate was statistically insignificant $(p>0.05)$ with respect to MELD scores. Probability of survival was 0.80 .

Conclusion: Liver cirrhosis with MELD score $\geq 18$ and variceal bleeding is highly prevalent in young adult patients, more likely in male patients having duration of disease since $>1$ year to 3 years such that every 1 of 5 patients expired during the hospital stay. Probability of survival was $80 \%$.
\end{abstract}

Key Words: Mortality, Cirrhotic patients, MELD (Model for End-stage Liver Disease) score, Variceal bleeding.

How to cite this article: Mehmood M, Zia MQ, Latif A, Ansar S. Mortality related factors in patients with variceal bleeding with MELD score $\geq 18$. J Coll Physicians Surg Pak 2019; 29(12):1199-1202.

\section{INTRODUCTION}

Cirrhosis is the end result of chronic liver damage caused by chronic liver diseases and defined as scarring of liver with poor liver functions. Common causes of chronic liver diseases include chronic hepatitis $B$ and $C$ and alcohol. ${ }^{1}$ However, the in-hospital mortality of cirrhotic patients was reported substantially high ranging from 35 to 90 percent. ${ }^{2}$ In Saudi Arabia, chronic liver disease and its complications constitute a major health problem and likely to increase in the years to come. ${ }^{3}$

Development of portal hypertension signifies the progression of liver cirrhosis and heralds the onset of fatal complications. Esophageal variceal bleeding is the

Correspondence: Dr. Tahir Mehmood, Department of Emergency

Medicine, Prince Sultan Military Medical City, Riyadh,

Saudi Arabia

E-mail:drtahirskt@yahoo.com

Received: May 03, 2018; Revised: May 22, 2019;

Accepted: June 19, 2019 life threatening complication of cirrhosis. Around onethird of cirrhotic patients will experience bleeding from esophageal or gastro-esophageal varices, with $70 \%$ recurrence rate and $20 \%$ mortality rate. ${ }^{4}$ The highest mortality peak is observed during the first 6 weeks after the bleeding episode. Increased mortality is associated with hepatic functional status, renal dysfunction and bacterial infections. 5,6

The Child-Turcotte-Pugh (CTP) classification serves as an easy to use prognostic marker in patients with variceal bleeding. However, two subjective variables (ascites and encephalopathy) can interfere with its accuracy and limits its application. Other markers of prognosis in patients with variceal bleeding include number of blood transfusions, platelet count and MELD (Model for Endstage Liver Disease) score. ${ }^{7}$

The MELD score, initially developed to assess prognosis following TIPS placement, is now widely accepted as a prognostic model in advanced cirrhosis. MELD score allows identifying patients at risk of short-term mortality 
among cirrhotic patients with bleeding from esophageal varices. In one study, there was $18.6 \%$ in-hospital mortality rate for cirrhotic patients with variceal bleeding with a MELD score of $\geq 18.5$ While another study revealed MELD score as a strong predictor of variceal rebleeding and increased risk of mortality after 6 weeks of acute variceal bleeding. 6

Esophageal variceal bleeding mortality predictors remains a major area of interest; and cutting edge research projects are underway as indicated by Baveno proceedings and the hope is to develop a consensus on a model for accurately predicting survival in cirrhotic patients with variceal bleeding. ${ }^{7}$

This study may be useful in evaluating the importance of MELD score for determination of risk of in-hospital mortality in cirrhotic patients with variceal bleeding. ${ }^{8}$ On admission, patient stratification into high and low mortality risk on the basis of MELD score can guide the initial level of patient care.

The aim of this study was to find associated determinants and patient prognosis in cirrhotic patients presenting with variceal bleeding having MELD score $\geq 18$.

\section{METHODOLOGY}

This descriptive cross-sectional study expanded over 6 months from July 2017 to January 2018. It included 235 patients recruited through non-probability consecutive sampling from the Emergency Department, King Sultan Military Hospital Riyadh, Kingdom of Saudi Arabia.

The target sample was calculated by using WHO sample size calculator, assuming anticipated percentage in-hospital mortality $18.6 \%$ in cirrhotic patients with MELD score $\geq 18$ presenting in Emergency Department with variceal bleeding; 5235 cases were required on $95 \%$ confidence level and $5 \%$ margin of error. All cirrhotic patients (>6 months) of both genders, 20-60 years of age presenting in emergency with first episode of variceal bleed having a MELD score $\geq 18$ were included; while patients were excluded, if they had either portosystemic encephalopathy (on clinical basis), upper gastrointestinal $(\mathrm{Gl})$ bleeding due to causes other than varices (seen on upper GI endoscopy), and with comorbid conditions like diabetes mellitus, hypertension or hepatocellular carcinoma.

Informed consent was taken in the language they understood the best. Patients were managed as per standard protocol of acute variceal bleeding. ${ }^{5}$ Blood samples were taken for complete blood counts, prothrombin time, liver function tests, renal function tests, and albumin, before the start of therapy.

Patients were diagnosed cirrhotic if they had $<150000 / \mu \mathrm{l}$, PT $>3$ sec prolonged), biochemical (reversal of ALT, AST ratio, albumin $<3.5 \mathrm{~g} / \mathrm{dl}$ ) and imaging studies (coarse echotexture of liver, splenomegaly) presence of all of the above and for more than 6 months. Variceal bleeding diagnosed on endoscopy. MELD score was calculated as MELD $=3.78$ [Ln serum bilirubin $(\mathrm{mg} / \mathrm{dL})]+11.2[\mathrm{Ln}$ INR] +9.57 [Ln serum creatinine $(\mathrm{mg} / \mathrm{dL})]+6.430 \mathrm{NR}$. Patients demographics like name, age, gender, address, were noted. The patient's in-hospital outcome was measured in terms of either alive or expired during hospital admission within 30 days.

Data analysis was performed by using the Statistical Software for Social Sciences (SPSS) version 20.0 (IBM, Chicago-USA). Frequencies with percentages were calculated for categorical variables like, age, duration of cirrhosis, Child Pugh class and MELD score. To find associated determinants of in-hospital mortality of cirrhotic patients with demographic features and MELD score, Chi-Square test was applied by taking in-hospital mortality as outcome of patients; while factor variables including demographic data and MELD score were stratified to measure any association with specific category as potential risk factor leading to in-hospital mortality. Survival analysis was performed to determine the probability of survival. P-value $\leq 0.05$ was considered statistically significant result.

\section{RESULTS}

To evaluated association of demographic features and MELD score with in-hospital mortality, binary logistic regression model was made by taking in-hospital mortality as binary outcome of patients; while factor variables, including demographic data and MELD score, were stratified to measure any association with specific category as potential risk factor leading to in-hospital mortality (Table I).

Among 235 patients with cirrhosis, 156 (66.4\%) were males and $79(33.6 \%)$ were females. The mean age of the patients was $47.8 \pm 8.7$ years. There were $82(34.9 \%)$ having Child Pugh score B and $153(65.1 \%)$ had Child Pugh score $C$.

Out of 235 patients of liver cirrhosis, $47(20 \%)$ expired during the hospital stay while $188(80 \%)$ patients survived and discharged from the hospital (Table II).

Table I: General demographic data.

\begin{tabular}{l|c|c}
\hline Total patients in the study & 235 & $100 \%$ \\
\hline Age & $35-53$ years & (mean $48 \pm 6$ years) \\
\hline Gender (m:f) & $156: 79$ & $(1.974: 1)$ \\
\hline Duration of cirrhosis & $3-6$ years & $4 \pm 1.2$ years \\
\hline Child-Pugh score & B-82 (34.9\%) & \\
\hline C-153 (65.1\%) & \\
\hline Hepatitis B & 19 & $8.08 \%$ \\
\hline
\end{tabular}

Table II: Morbidity and mortality.

\begin{tabular}{l|c|c}
\hline Prolonged hospital stay & 146 & $62.12 \%$ \\
\hline Encephalopathy & 39 & $16.59 \%$ \\
\hline Re-bleed & 12 & $5.10 \%$ \\
\hline Mortality & 47 & $20.42 \%$ \\
\hline
\end{tabular}




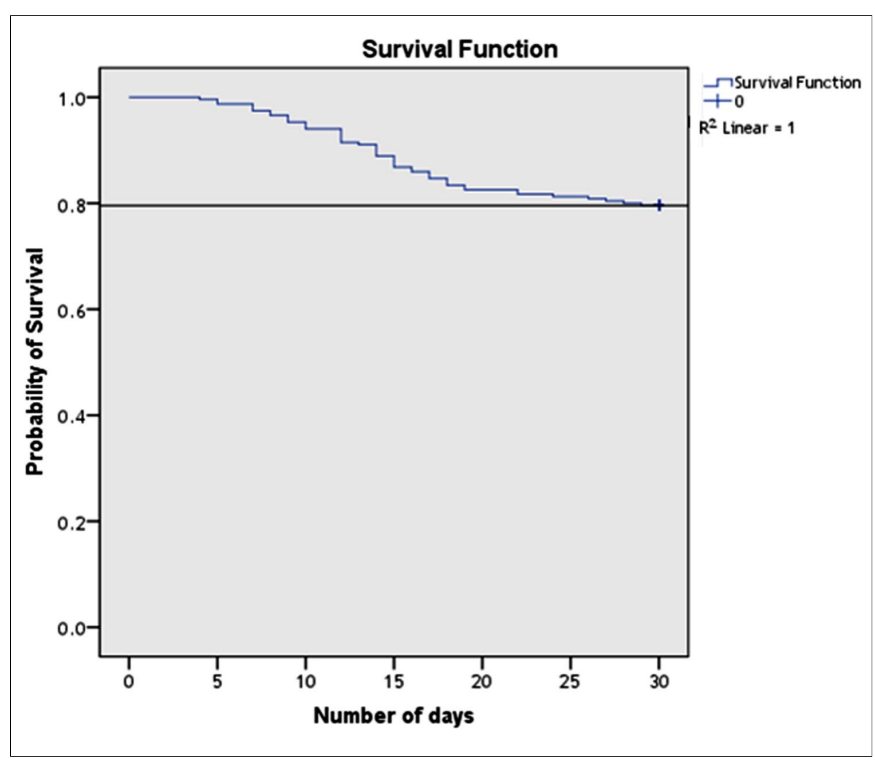

Figure 1: Probability of survival $=0.80$.

Out of 156 male patients, $34(21.8 \%)$ expired during the hospital stay; while among 79 female patients, 13 (16.5\%) died. The mortality rate was not statistically significant between male \& female patients $(O R=0.68, p=0.284)$. Most of the cirrhotic patients were in the age brackets of 41-50 years, i.e. 97 (41.3\%) patients; and 91 (38.7\%) in 51-60 years. However, in-hospital mortality of cirrhotic patient was not found associated with some specific age group. Most of the patients had cirrhosis for $>1$ year to 3 years, i.e. $86(36.6 \%)$ had $13-24$ months and 81 $(34.5 \%)$ had duration of cirrhosis since $25-36$ months.

Out of 82 patients of child pugh class B of liver cirrhosis, $14(17.1 \%)$ died in hospital; and out of 153 patients of child pugh class C, 34 (22.2\%) expired during hospital stay. Most of the cirrhotic patients were experienced with MELD score $18-20$, i.e. 144 (61.3\%) followed by 70 $(29.8 \%)$ in $21-25$ and 21 (8.9\%) had the range of $26-30$. Probability of survival was found to be 0.80 revealing $20 \%$ risk of in-hospital mortality (Figure 1 ).

\section{DISCUSSION}

The principal finding of the study was found that liver cirrhosis with MELD score $\geq 18$ and variceal bleeding is highly prevalent in young adult patients such that every 1 of 5 patients (i.e. $20.4 \%$ ) expired during the hospital stay. As compared with the study of Cerqueira et al., 9 the in-hospital mortality was $18.6 \%$ for cirrhotic patients with variceal bleeding with a MELD score of $\geq 18$, which is comparable with our study; whereas, Afessa and Kubilis in the year 2000 reported an in-hospital mortality rate of $21 \%$ in bleeding cirrhotic. 10 Pauwels et al. showed that in-hospital mortality in cirrhotic patients admitted with variceal bleeding has decreased by $50 \%$ over the past 15 years. ${ }^{11}$ In 1986, Chojkier and colleagues reported a bleeding-related mortality rate of $35 \% .12$

In this study, the mean age of the patients was $47.0 \pm 8.5$ years. As compared with the study of Abdel-Aziz et al., ${ }^{13}$ the mean age of the patients was $52.0 \pm 4.2$ years, which is comparable with this study. According to the study conducted by Morsy et al., the mean age of the patients was $53.3 \pm 11.2$ years, ${ }^{14}$ which is also comparable with this study.

Shukla et al. reported that patients who were younger, with ascites, larger medical comorbidity, and higher MELD scores, had a higher risk of variceal bleeding. ${ }^{15}$ Most of the demographic and clinical factors were found associated with the risk of variceal bleeding. Compared with patients older than 65 years, the risk of bleeding was higher for <50-year and 50-64-year patients (adjusted $\mathrm{HR}=1.92,95 \% \mathrm{Cl}=1.36-2.72$ and $1.36,95 \%$ $\mathrm{Cl}=1.05-1.77$, respectively).

More recently, Chalasani et al. in a large study over three years reported that the in-hospital mortality to be $14.2 \% .{ }^{16}$ In another large series of 403 patients with liver cirrhosis and variceal bleeding, Del Olmo and colleagues reported a mortality rate of $7.4 \% .{ }^{17}$ Similar decline in hospital mortality has been reported in other studies, too.

In this study, $66.4 \%$ patients were males and $33.6 \%$ patients were females. As compared with the study of Morsy et al.,14 $65.6 \%$ patients were males and $34.4 \%$ patients were females which is same and comparable with this study. In another study conducted by Abdel-Aziz et al.,13 there were $55.9 \%$ males and $44.1 \%$ females, which is also comparable with this study.

In this study, $34.9 \%$ patients had Child Pugh score B and $35.1 \%$ patients had Child Pugh score $\mathrm{C}$. As compared with the study of Morsy et al.,14 $12.9 \%$ patients had Child Pugh score $B$.

The recently developed modified score for critically ill cirrhosis $(\mathrm{MSClC})$ yielded outstanding predictive value (probability under the receiver operating curve: $0.856 \pm$ 0.047 ), discriminative and calibration ability similar to the MELD. 18

The limitation of our study was the predictive accuracy of the MELD score; and the prognosis could not be exhibited. However, this study provides baseline data of in-hospital mortality of patients with MELD score $\geq 18$.

\section{CONCLUSION}

Liver cirrhosis with MELD score $>18$ and variceal bleeding is highly prevalent in young adult patients, more likely in male patients having duration of disease since $>1$ year to 3 years such that every 1 of 5 patients expired during the hospital stay. Probability of survival was $80 \%$.

\section{ETHICAL APPROVAL:}

Research Ethics Committee of Prince Sultan Military Medical City approved the study.

\section{PATIENTS' CONSENT:}

Informed consents were taken in the language the patients understood the best. 


\section{CONFLICT OF INTEREST:}

Authors declared no conflict of interest.

\section{AUTHORS' CONTRIBUTION:}

TM: Study design, statistical analysis; data collection. MQZ: Concept, search of literature, critical revision, manuscript writing, proofreading.

AL: Statistical analysis, major changes, manuscritp writing, proofreading, final approval for publication.

SA: Search of literature.

\section{REFERENCES}

1. Lavanchy D. The global burden of hepatitis C. Liver int 2009; 29: 74-81.

2. Levesque E, Saliba F, Ichai P, Samuel D. Outcome of patients with cirrhosis requiring mechanical ventilation in ICU. $J$ Hepatol 2014; 60:570-8.

3. Shobokshi OA, Serebour FE, Al-Drees AZ, Mitwalli AH, Qahtani A, Skakni LI. Hepatitis C virus seroprevalence rate among Saudis. Saudi Med J 2003; 24:81-6.

4. Bosch J, Berzigotti A, Garcia-Pagan JC, Abraldes JG. The management of portal hypertension: Rational basis, available treatments and future options. J Hepatol 2008; 48(Suppl 1):S68-92.

5. Cerqueira RM, Andrade L, Correia MR, Fernandes CD, Manso MC. Risk factors for in-hospital mortality in cirrhotic patients with oesophageal variceal bleeding. Eur J Gastroenterol Hepatol 2012; 24:551-7.

6. Bambha K, Kim WR, Pedersen R, Bida JP, Kremers WK, Kamath PS. Predictors of early rebleeding and mortality after acute variceal bleeding in patients with chirrosis. Gut 2008; 57:814-17.

7. De Franchis R. Revising consensus in portal hypertension: Report of the Baveno $\mathrm{V}$ consensus workshop on methodology of diagnosis and therapy in portal hypertension. J Hepatol 2010; 53:762-8.

8. Reverter E, Tandon P, Augustin S, Turon F, Casu S, Bastiampillai $\mathrm{R}$, et al. A MELD-based model to determine risk of mortality among patients with acute variceal bleeding. Gastroenterology 2014; 146:412-19.

9. Cerqueira RM, Andrade L, Correia MR, Fernandes CD, Manso MC. Risk factors for in-hospital mortality in cirrhotic patients with oesophageal variceal bleeding. Eur J Gastroenterol Hepatol 2012; 24:551-7.

10. Afessa B, Kubilis PS. Upper gastrointestinal bleeding in patients with hepatic cirrhosis: clinical course and mortality prediction. Am J Gastroenterol 2000; 95:484-9.

11. Pauwels A, Fourdan O, Carbonell N. Mortality from digestion hemorrhage for the last fifteen years. Gastroenterol Clin Biol 1998; 22(Suppl 2):A27.

12. Chojkier M, Laine L, Conn HO, Lerner E. Predictors of outcome in massive upper gastrointestinal hemorrhage. J Clin Gastroenterol 1986; 8:16-22.

13. Abdel-Aziz IM, Haraf TM, Rashad FE. Factors predicting in hospital mortality in patients with liver cirrhosis hospitalized with gastroesophageal variceal hemorrhage. AAMJ 2008; 6:421-5.

14. Morsy KH, Ghaliony MAA, Mohammed HS. Outcomes and predictors of in-hospital mortality among cirrhotic patients with non-variceal upper gastrointestinal bleeding in upper Egypt. Turk J Gastroenterol 2014; 25:707-13.

15. Shukla R, Kramer J, Cao Y, Ying J, Tansel A, Walder A, et al. Risk and predictors of variceal bleeding in cirrhosis patients receiving primary prophylaxis with non-selective beta-blockers. Am J Gastroenterol 2016; 111:1778-8.

16. Chalsani N, Kahi C, Francois F, Pinto A, Marathe A, Bini J, et al. Improved patient survival after acute var iceal bleeding: A multicenter, cohort study. Am J Gatstroenterol 2003; 98:653-9.

17. Del Olmo JA, Pena A, Serra MA, Wassel AH, Benages A, Rodrigo JM. Predictors of morbidity and mortality after the first episode of upper gastrointestinal bleeding in liver cirrhosis. J Hepatol 2000; 32:19-24.

18. Bao $Q$, Wang $B$, Liang $Y u$, Weng $H$, Jianping Ge, Lanjuan Li. A modified prognostic score for critically ill patients with cirrhosis: An observational study. J Gastroenterol Hepatol 2016; 31:450-8. 